PROCEEDINGS OF THE

AMERICAN MATHEMATICAL SOCIETY

Volume 126, Number 10, October 1998, Pages 2905-2908

S $0002-9939(98) 04529-8$

\title{
NOTE ON THE TOPOLOGICAL DEGREE OF THE SUBDIFFERENTIAL OF A LOWER SEMI-CONTINUOUS CONVEX FUNCTION
}

\author{
SERGIU AIZICOVICI AND YUQING CHEN \\ (Communicated by Palle E. T. Jorgensen)
}

\begin{abstract}
The purpose of the present paper is to prove that the topological degree of the subdifferential of a coercive lower semi-continuous function on a sufficiently large ball in a reflexive Banach space is equal to one.
\end{abstract}

A well known result of Krasnoselskii [7] says that the degree of the gradient of a coercive potential mapping on a sufficiently large ball in $R^{N}$ is equal to one. As Nirenberg pointed out in [8], this result seems intuitively clear, but the proof is not. Many authors had new views of this theorem. See [1], [9]-[11]. The aim of this note is to give a generalization of the degree of a maximal monotone mapping and prove that the topological degree of the subdifferential of a coercive convex function is equal to one on a sufficiently large ball. The main result of this paper is Theorem 5 . Its proof is very simple and elementary.

Let us recall the properties of the degree of mappings of class $\left(S_{+}\right)$, as introduced by Browder [4]. See also [3], [6]. Let $E$ be a real reflexive Banach space of dual $E^{*}$. We can always assume that both $E$ and $E^{*}$ are locally uniformly convex [12]. The duality pairing between $E^{*}$ and $E$ is denoted by $(.,$.$) . Let \theta^{*}$ be the zero element of $E^{*}, J: E \rightarrow E^{*}$ the duality mapping, $\Omega \subset E$ an open bounded subset, and $T: \bar{\Omega} \rightarrow E^{*}$ a demi-continuous operator of class $\left(S_{+}\right)$(see [4] for a definition); if $\theta^{*} \notin T(\partial \Omega)$, then there is a topological degree $\operatorname{deg}\left(T, \Omega, \theta^{*}\right)$ satisfying

Theorem 1. (a)

$$
\operatorname{deg}\left(J, \Omega, \theta^{*}\right)=\left\{\begin{array}{l}
1, \text { if } \theta^{*} \in J(\Omega), \\
0, \text { if } \theta^{*} \notin J(\bar{\Omega}) .
\end{array}\right.
$$

(b) If $\operatorname{deg}\left(T, \Omega, \theta^{*}\right) \neq 0$, then $T x=\theta^{*}$ has a solution in $\Omega$.

(c) If $\Omega_{1}, \Omega_{2}$ are two disjoint open subsets of $\Omega$, then

$$
\operatorname{deg}\left(T, \Omega_{1}, \theta^{*}\right)+\operatorname{deg}\left(T, \Omega_{2}, \theta^{*}\right)=\operatorname{deg}\left(T, \Omega_{1} \cup \Omega_{2}, \theta^{*}\right) .
$$

(d) If $\left\{T_{t}\right\}_{[0,1]}$ is a homotopy of class $\left(S_{+}\right)$, and $\theta^{*} \notin T_{t}(\partial \Omega)$, then $\operatorname{deg}\left(T, \Omega, \theta^{*}\right)$ doesn't depend on $t \in[0,1]$.

Now, suppose that $A: D(A) \subseteq E \rightarrow 2^{E^{*}}$ is a maximal monotone operator. Let $A_{\lambda}=\left(\lambda J^{-1}+A^{-1}\right)^{-1}, R_{\lambda}=I-\lambda J^{-1} A_{\lambda}$ be its Yosida approximation and resolvent

Received by the editors February 25, 1997.

1991 Mathematics Subject Classification. Primary 47H10, 47H15; Secondary 55M25.

Key words and phrases. Monotone operator, class $\left(S_{+}\right)$, topological degree.

(C)1998 American Mathematical Society 
respectively, and $T: E \rightarrow E^{*}$ be a demi-continuous bounded operator of class $\left(S_{+}\right)$. We introduce $\operatorname{deg}\left(A, D(A) \cap \Omega, \theta^{*}\right)$ by means of several lemmas. In what follows " $\rightarrow$ " stands for weak convergence.

Lemma 2. If $x_{j} \in D(A)$, with $x_{j} \rightarrow x_{0}$, and $\epsilon_{j}$ is a positive sequence converging to 0 such that $\theta^{*} \in\left(A+\epsilon_{j} T\right) x_{j}$, then $x_{j} \rightarrow x_{0}$, and $\theta^{*} \in A x_{0}$.

Proof. By assumption, we have

$$
\left(\epsilon_{j} T x_{j}-\epsilon_{i} T x_{i}, x_{j}-x_{i}\right) \leq 0, \forall i, j \geq 1 .
$$

Letting $i \rightarrow 0$, we get

$$
\epsilon_{j}\left(T x_{j}, x_{j}-x_{0}\right) \leq 0 .
$$

But $T$ is an operator of class $\left(S_{+}\right)$, so $x_{j} \rightarrow x_{0}$, and $T x_{j} \rightarrow T x_{0}$. Hence, we get $x_{0} \in D(A)$, and $\theta^{*} \in A x_{0}$. This completes the proof.

Now, suppose that $\Omega \cap D(A) \neq \varnothing$, and $\theta^{*} \notin A x, \forall x \in \partial \Omega \cap D(A)$; then by using Lemma 2, we know that there exists $\epsilon_{0}>0$, such that $\theta^{*} \notin(A+\epsilon T)(\partial \Omega)$ for $\epsilon \in\left(0, \epsilon_{0}\right)$. By adapting the technique in [4], one can show that there exists $\lambda_{0}(\epsilon)>0$, such that

$$
\theta^{*} \notin\left(A_{\lambda}+\epsilon T\right)(\partial \Omega), \forall \lambda \in\left(0, \lambda_{0}(\epsilon)\right) .
$$

Note that $A_{\lambda}+\epsilon T$ is a demi-continuous operator of class $\left(S_{+}\right)$, so that the degree $\operatorname{deg}\left(A_{\lambda}+\epsilon T, \Omega, \theta^{*}\right)$ is well defined. We show that this degree doesn't depend on $\epsilon, \lambda$, and $T$. Specifically we have:

Lemma 3. Let $T_{1}, T_{2}$ be two demi-continuous operators of class $\left(S_{+}\right)$, and $\lambda_{1}, \lambda_{2}$ $>0, \epsilon_{1}, \epsilon_{2}>0$. Then $\left\{A_{t \lambda_{1}+(1-t) \lambda_{2}}+t \epsilon_{1} T_{1}+(1-t) \epsilon_{2} T_{2}\right\}_{t \in[0,1]}$ is a homotopy of class $\left(S_{+}\right)$.

The proof of this lemma relies on the following result.

Lemma 4 (cf. Proposition 3.56 in [2]). If $\lambda_{j} \rightarrow \lambda$, then $A_{\lambda_{j}} x \rightarrow A_{\lambda} x$, for any $x \in E$.

Proof of Lemma 3. Let $t_{j} \rightarrow t_{0}, x_{j} \rightarrow x_{0}$ be such that

$$
\varlimsup_{j \rightarrow \infty}\left(A_{t_{j} \lambda_{1}+\left(1-t_{j}\right) \lambda_{2}} x_{j}+t_{j} \epsilon_{1} T_{1} x_{j}+\left(1-t_{j}\right) \epsilon_{2} T_{2} x_{j}, x_{j}-x_{0}\right) \leq 0 .
$$

By the monotonicity of $A_{\lambda}$ and Lemma 4 , we know that

$$
\underline{\lim }_{j \rightarrow \infty}\left(A_{t_{j} \lambda_{1}+\left(1-t_{j}\right) \lambda_{2}} x_{j}, x_{j}-x_{0}\right) \geq 0 .
$$

Consequently

$$
\overline{\lim }_{j \rightarrow \infty}\left(t_{j} \epsilon_{1} T_{1} x_{j}+\left(1-t_{j}\right) \epsilon_{2} T_{2} x_{j}, x_{j}-x_{0}\right) \leq 0 .
$$

Since $\left\{t \epsilon_{1} T_{1}+(1-t) \epsilon_{2} T_{2}\right\}_{t \in[0,1]}$ is a homotopy of class $\left(S_{+}\right)$(see [4]), it follows that $x_{j} \rightarrow x_{0}, T_{1} x_{j} \rightarrow T_{1} x_{0}, T_{2} x_{j} \rightarrow T_{2} x_{0}, A_{t_{j} \lambda_{1}+\left(1-t_{j}\right) \lambda_{2}} x_{j} \rightarrow A_{t_{0} \lambda_{1}+\left(1-t_{0}\right) \lambda_{2}} x_{0}$. The proof is complete.

The above arguments show that $\operatorname{deg}\left(A_{\lambda}+\epsilon T, \Omega, \theta^{*}\right)$ is constant for sufficiently small $\epsilon, \lambda>0$ and doesn't depend on $T$, so we can define the topological degree $\operatorname{deg}\left(A, D(A) \cap \Omega, \theta^{*}\right)$ as the common value of $\operatorname{deg}\left(A_{\lambda}+\epsilon T, \Omega, \theta^{*}\right)$. In particular the theory applies to the case when $A=\partial \varphi$, the subdifferential of a proper, convex, and lower semi-continuous function $\varphi: E \rightarrow(-\infty,+\infty]$. 
Our main result is the following

Theorem 5. Let $\varphi: D(\varphi) \subseteq E \rightarrow(-\infty,+\infty]$ be a proper lower-semicontinuous convex function such that $\lim _{\|x\| \rightarrow \infty} \varphi(x)=\infty$. Then there exists $r_{0}>0$, such that

$$
\operatorname{deg}\left(\partial \varphi, D(\partial \varphi) \cap B(0, r), \theta^{*}\right)=1
$$

for any $r>r_{0}$.

Proof. Since $\lim _{\|x\| \rightarrow \infty} \varphi(x)=+\infty$, there exists $x_{0} \in E$, such that $\theta^{*} \in \partial \varphi\left(x_{0}\right)$. Let $r_{0}>0$ be sufficiently large such that $\theta^{*} \notin \partial \varphi(x)$ for any $x \in D(\partial \varphi) \cap \partial B(0, r)$, and any $r \geq r_{0}$.

There exists an $\epsilon_{0}>0$, such that

$$
\begin{aligned}
& \theta^{*} \notin \cup_{t \in[0,1]}\left(t \partial \varphi(x)+t \epsilon J\left(x-x_{0}\right)+(1-t) \epsilon J\left(x-x_{0}\right)\right) \\
& \forall x \in D(\partial \varphi) \cap \partial B(0, r), \epsilon \in\left(0, \epsilon_{0}\right) .
\end{aligned}
$$

For each $\epsilon \in\left(0, \epsilon_{0}\right)$, one can prove by contradiction that there exists $\lambda_{0}>0$ with the property

$$
\theta^{*} \notin \cup_{t \in[0,1]}\left(t \partial \varphi_{\lambda}+t \epsilon J\left(.-x_{0}\right)+(1-t) \epsilon J\left(.-x_{0}\right)\right) \partial B(0, r), \forall \lambda \in\left(0, \lambda_{0}\right) .
$$

By (a), (d) of Theorem 1, we know that

$$
\operatorname{deg}\left(\partial \varphi_{\lambda}+\epsilon J\left(.-x_{0}\right), B(0, r), \theta^{*}\right)=\operatorname{deg}\left(J\left(.-x_{0}\right), B(0, r), \theta^{*}\right)=1 .
$$

So $\operatorname{deg}\left(\partial \varphi, D(\partial \varphi \cap B(0, r)), \theta^{*}\right)=1$, as desired.

A more general form of Theorem 5 is

Theorem 6. Let $A: D(A) \subseteq E \rightarrow 2^{E^{*}}$ be a maximal monotone operator, and $\Omega \subset E$ be an open bounded subset with $\Omega \cap D(A) \neq \varnothing$. Suppose that there exists $\bar{x} \in \Omega \cap D(A)$, such that $(f, x-\bar{x}) \geq 0, \forall x \in D(A) \cap \partial \Omega, f \in A x$, and $\theta^{*} \notin A x, \forall x \in$ $D(A) \cap \partial \Omega$. Then $\operatorname{deg}\left(A, \Omega \cap D(A), \theta^{*}\right)=1$.

The proof of this theorem is similar to that of Theorem 5, and we omit the details.

\section{REFERENCES}

[1] H. Amann, A note on degree theory for gradient mappings, Proc. Amer. Math. Soc., 85(1982),591-595. MR 83i:47069

[2] H. Attouch, Variational Convergence for Functions and Operators, Pitman,Boston,1984. MR 86f: 49002

[3] J. Berkovits and V. Mustonen, On the degree for mappings of monotone type, Nonlinear Anal., 12(1986),1373-1383. MR 88b:47073

[4] F.E.Browder, Fixed point theory and nonlinear problems, Bull.Amer.Math. Soc., 1(1983),139. MR 84h:58027

[5] F.E.Browder, Degree theory for nonlinear mappings, Proc. Symp.Pure Math., Vol.45,pp.203226, Amer.Math.Soc., Providence,R.I., 1986. MR 87g:47108

[6] Y.Q. Chen and S. S. Zhang, Degree theory for multivalued (S) type mappings and fixed point theorems, Applied Math.Mech., English Ed., 11(1990),441-454. MR 91h:47065

[7] M.A. Krasnoselskii, Topological Methods in the Theory of Nonlinear Integral Equations,Macmillan, New York,1964. MR 28:2414

[8] L. Nirenberg, Variational and topological methods in nonlinear problems, Bull. Amer. Math. Soc., 4(1981),267-302. MR 83e:58015

[9] P. H. Rabinowitz, A note on topological degree for potential operators, J. Math. Anal. Appl., 51(1975),483-492. MR 57:10518 
[10] E. H. Rothe, A relation between the type numbers of a critical point and the index of the corresponding field of gradient vectors, Math. Nachr., 4(1950-51), 12-17. MR 12:720c

[11] J. C. Scovel, A simple intuitive proof of a theorem in degree theory for gradient mappings, Proc. Amer. Math. Soc., 93(1985), 751-753. MR 86g:55001

[12] S. L. Trojansky, On locally uniformly convex and differentiable norms in certain nonseparable Banach spaces, Studia Math., 37(1971), 173-180.

Department of Mathematics, Ohio University, Athens, Ohio 45701-2979

E-mail address: aizicovi@bing.math.ohiou.edu

E-mail address: yuqchen@bing.math.ohiou.edu 